\title{
Nonlinear signal processing, spectral, and fractal-based stridor auscultation: A machine learning approach
}

\author{
Vimal Raj ${ }^{1}$, A. Renjini ${ }^{2}$, M. S. Swapna ${ }^{3}$, S. Sreejyothi ${ }^{4}$, and S. Sankararaman* \\ Dept. of Optoelectronics, University of Kerala, Trivandrum, \\ Kerala, India- 695581 \\ *corresponding author: drssraman@gmail.com
}

\begin{abstract}
The work reported in the paper analyses the adventitious stridor breath sound (ST) and the normal bronchial breath sound (BR) using spectral, fractal, and nonlinear signal processing methods. The sixty breath sound signals are subjected to power spectral density (PSD) and wavelet analyses to understand the temporal evolution of the frequency components. The energy envelope of the PSD plot of ST shows three peaks labeled as A $(256 \mathrm{~Hz}), \mathrm{B}(369 \mathrm{~Hz})$, and C $(540 \mathrm{~Hz})$, of which A alone is present in BR at $265 \mathrm{~Hz}$. The appearance of B and C in the PSD plot of ST is due to the obstructions in the trachea and upper airways caused by lesions. The phase portrait analysis of the time series data of ST and BR gives information about the dynamical system's randomness and sample entropy. The study reveals that the fractal dimension and sample entropy values are higher for BR, which may be due to the musical ordered behavior of ST. The machine learning techniques based on the features extracted from the PSD data and phase portrait parameters offer good predictability, besides the classification of BR and ST, thereby revealing its potential in pulmonary auscultation.
\end{abstract}

Keywords: Breath sound; fractal; linear discriminant analysis; nonlinear time series; stridor.

\section{Introduction}

Our environment is the storehouse of essential elements for our living and harmful microorganisms like viruses, fungi, and bacteria. These microorganisms and aerosol pollutants cause chronic respiratory tract diseases in humans and other living organisms (Monto \& Fukuda, 2020). Asthma, tuberculosis, lung cancer, chronic obstructive pulmonary disease, and acute lower respiratory tract infections are the five respiratory tract diseases among the top most common causes of death ( $\mathrm{Li}$ et al., 2020). Therefore, there is a need for accurate and earlier detection methods to decrease the number of deaths and health burdens caused by respiratory tract diseases. Breath sounds heard at specific locations on the respiratory tract carry information about various health conditions (Gavriely et al., 1981; Debbal \& Bereksi-Reguig, 2008). The friction between the airway walls and the turbulent airflow in the respiratory tract during respiration is the primary cause of breath sounds. In addition, the glottis and airflow from a narrow to a broader region also produce breath sounds (Jones, 1995). Auscultation is the primary method that analyses breath sounds to diagnose and monitor different respiratory tract diseases. The main two groups of breath sounds are normal and adventitious breath sounds (Bohadana et al., 2014). 
Normal breath sounds like vesicular and bronchial breath sounds are heard over the respiratory tract during normal functioning. The bronchial breath sounds (BR) are auscultated over the large airways - trachea (Bohadana et al., 2014; Andrès et al., 2018). The bronchial sounds are louder and high-pitched relative to the vesicular breaths sounds auscultated over the lung periphery. The respiratory tract problems like infection, obstruction, inflammation, asthma and others perturb the normal breath sounds and become adventitious. Crackles, stridor, pleural rub, rhonchi, and wheezing are the familiar adventitious breath sounds (Sarkar et al., 2015; Pramono et al., 2017). Among the adventitious signals, stridor breath sound (ST) is a highpitched, musical sound formed due to the turbulent airflow through the narrowed segment of the upper airways of the respiratory tract like trachea, glottis, subglottis, or supraglottis. Stridor sound can be heard during inspiration, expiration, or respiration (biphasic). Stridor sound is heard while inspiration due to the upper airway obstruction caused by the extrathoracic laryngomalacia and vocal cord lesion. Due to the intrathoracic tracheomalacia and bronchomalacia lesions, the upper airway obstruction is responsible for the stridor sound during expiration. Stridor breath sounds heard during respiration (both inspiration and expiration biphasic) are due to the upper airway obstruction due to fixed lesions such as laryngeal mass or web, croup, and others. Stridor breath sounds can be auscultated over the upper airways or even be heard without a stethoscope (Pfleger \& Eber, 2016; Udupi \& Prabhakar, 2016).

Stridor breath sounds can occur at any age. The presence of ST is more in children because of their softer and narrow respiratory tract (Gray et al., 1985; Leiberman et al., 1986). The removal of the tracheal tube (extubation) from patients in ventilator care can damage the upper airways, resulting in the development of ST (Pluijms et al., 2015). Thus, the ST is an indicator of several respiratory conditions that leads to upper airway obstruction in the respiratory tract. The analysis of breath sounds like ST using computer-aided digital techniques, which eliminates the shortcomings of the conventional auscultation like inter listener variability, could make the auscultation accurate, faster, and automatic (Debbal \& Bereksi-Reguig, 2008; Gurung et al., 2011). Breath sounds are nonstationary time series signals formed by the pressure variations due to the passage of turbulent air through the respiratory tract (Bohadana et al., 2014). The pressure variations contain several frequency components related to the mechanism of sound production, which can be studied using the Fourier transform technique employing a Fast Fourier transform (FFT) algorithm. The FFT analysis only gives information about the frequency components present in the signal but not about their temporal details. The time of occurrence of a particular frequency and its temporal evolution can be studied using wavelet analysis (Gavriely et al., 1981; Polat \& Guler, 2004; Debbal \& Bereksi-Reguig, 2008). The turbulent airflow through the respiratory tract can be considered a dynamic system.

Nonlinear time series analysis is a proven nonlinear signal processing tool to unveil the underlying dynamics using phase portrait, maximal Lyapunov exponent, and sample entropy. The phase portrait analysis reconstructs the attractor of the dynamical system using the time lag and embedding dimension, which gives a geometrical representation of the system's dynamics. Maximal Lyapunov exponent of a system measures the divergence of two attractor points with time, thus providing information about the degree of predictability. The sample entropy quantifies the disorder or uncertainty of a time series, thereby giving information about the complexity of the underlying dynamical system (Kantz \& Schreiber, 2003; Raj et al., 2020). 
The self-affine nature and complexity of the breath sound signals can be probed and quantified by estimating the fractal dimension through fractal analysis (Kenkel \& Walker, 1996). The boxcounting method, which counts the number of boxes to fill the pattern at different box sizes, is an easy and effective method for estimating the fractal dimension (Swapna et al., 2020). Hurst exponent, calculated from the fractal dimension, classifies the signal based on its nature of randomness (García \& Requena, 2019).

Automatic and fast diagnosis of disease from different biomedical signals like a heartbeat, lung sounds, electrocardiogram (ECG), and others using machine learning techniques (MLTs) is now essential for clinical monitoring. The MLTs rely on statistics and learning algorithms to develop models to classify and cluster data. The two machine learning approaches for biomedical signals are supervised and unsupervised. The supervised approach learns the similarity within the classes and boundaries between the types from labeled datasets and then predicts new input datasets. However, in the unsupervised approach, the machine groups or clusters the unlabelled data by finding the similarities and boundaries using statistical theories. The unsupervised principal component analysis (PCA) is a linear transformation tool based on dimensionality reduction used for grouping breath sound signals without labeling the dataset (Jolliffe \& Cadima, 2016; Husson et al., 2017). The commonly used supervised MLTs for the classification of biomedical signals are Linear discriminant analysis (LDA), Quadratic discriminant analysis (QDA), support vector machines (SVMs), and k-nearest neighbor (KNN).

The Linear discriminant analysis (LDA) is a multivariate classification and dimensionality reduction technique. Unlike PCA, LDA maximizes the separability between the classes, using a linear decision boundary (Wallace et al., 1997). The QDA is another MLT similar to the LDA but with a quadratic border. The SVM classifies the classes by constructing linear hyperplanes that create maximum separation in higher dimensional spaces (Colak et al., 2016). The original data is nonlinearly mapped into higher dimensions using appropriate kernel functions like linear, quadratic, cubic, fine Gaussian, medium Gaussian, and coarse Gaussian. KNN is a robust and straightforward non-parametric lazy learning algorithm-based MLT for nonlinear systems. The KNN algorithm searches for courses with majority neighbors among the $\mathrm{k}$ nearest neighbors to classify a data point to a class. There are different types of KNN based on the kvalue, distance metric, and weight of the neighboring points (Noble, 2006; Subasi, 2019; M. S. Swapna et al., 2021). The present study aims to develop novel digital auscultation techniques for ST breath sounds, integrating the principles of mathematics, statistics, and computer-aided machine learning.

\section{Materials and methods}

The progress in biomedical signal processing has given us many tools for the rapid and accurate analysis of biomedical signals like electrocardiograms, electroencephalograms, breath sounds, and others. Digital audio signals of sixty ST (30) and BR (30) from various sound repositories are investigated in the present work (Easyauscultation, 2021; EMTprep, 2021; Medzcool, 2021). The time-domain signal is generated from the digital data using which its wavelet and fast Fourier transform are found for unveiling the nature and peculiarities of the path of airflow through the respiratory tract. The time-domain signal $\mathrm{x}(\mathrm{t})$ at a time $\mathrm{t}$ can be converted into 
Fourier transformed complex signal X(f) of frequency f using Equation (1) (Debbal \& BereksiReguig, 2008).

$X(f)=\int x(t) e^{i 2 \pi f t} d t$

The power spectral density (PSD) function, proportional to the square of the magnitude of the Fourier transformed signal $\mathrm{X}(\mathrm{f})$, gives the power distribution over its frequency components. The PSD function of a signal of length N, given by Equation (2), can be used to study the presence of a frequency component, its spread, and magnitude (Bahoura, 2009). The spectral signatures of various signals estimated using the PSD function could help classify signals. However, the temporal characteristics of the frequency component cannot be studied using the PSD function, which is overcome through wavelet analysis.

$P S D(f)=\frac{|X(f)|^{2}}{N}$,

Wavelet analysis employs a fixed-function called mother wavelet $(\varphi)$, translated through the time series signal to generate the wavelets. The mother wavelet function used in the present study is the Morse wavelet, suitable for time-varying amplitude and frequency signals. The wavelet analysis could estimate the dominant frequency and its magnitude at a particular instant (Debbal \& Bereksi-Reguig, 2008). The wavelet transform of a continuous time-series signal is given by Equation (3).

$W_{c} f(s, \tau)=\int_{-\infty}^{\infty} x(t) \cdot s^{-1 / 2} \varphi\left(\frac{t-\tau}{s}\right) d t$

where s and $\tau$ are the scale and translation parameters, respectively.

The turbulent airflow through the respiratory tract makes the breath sound nonlinear. Nonlinear time series analysis is a proven tool to unveil the nonlinearity in the data, which reflects the complexity of the underlying dynamics. The phase portrait gives the visual representation of the system's dynamics in the phase plane. The parameters time lag and embedding dimension are required to reconstruct phase portrait from the time series, which can be computed using the R software. Time lag $(l)$ is the time separation between the uncorrelated data points, and embedding dimension $(\mathrm{m})$ is the number of independent coordinates required for specifying a complex system. According to the method of delays, using the values of $l$ and $\mathrm{m}$ for a time series $\left(\mathrm{x}_{\mathrm{n}}\right)$, the phase portrait can be drawn using the vector given in Equation (4) (Kantz \& Schreiber, 2003).

$\boldsymbol{x}_{\mathbf{n}}=\left(x_{n-(m-1) l}, x_{(n-(m-2) l)}, \ldots \ldots, x_{n}\right)$

$\mathrm{R}$ software is employed for finding the coordinates of the phase portrait. The time lag, embedding dimension, and coordinates of the phase portrait are estimated using functions in the 'nonlinear series' package. The 'time-lag' function employing the nonlinear average mutual information function algorithm finds the time lag. The 'estimatingEmbeddingDim' function employing Cao's algorithm finds the embedding dimension (Cao, 1997). From the value of $l$ and $\mathrm{m}$, the coordinates of phase portrait are generated according to Equation (4), employing the 'buildTakens' function. 
The complexity of the dynamics of a nonlinear system can also be studied by estimating the maximal Lyapunov exponent ( $\lambda$ ) (Ahlstrom et al., 2006). The value of $\lambda$, pointing to the system's complexity, measures average exponential variation in the separation between two neighboring phase points in a phase portrait. If there is a divergence between the trajectories, the value of $\lambda$ is positive, indicating the system's sensitivity to the initial condition. The negative value of $\lambda$ points to convergence between the trajectories. The separation between two-phase points at time $\mathrm{t}\left(\delta \mathrm{Z}_{\mathrm{t}}\right)$ and $\mathrm{t}=0\left(\delta Z_{0}\right)$ are related, as given in Equation (5) (Kantz \& Schreiber, 2003).

$\left|\delta Z_{t}\right| \approx e^{\lambda t}\left|\delta Z_{0}\right|$

The slope of $\log \frac{\delta \mathrm{Z}_{t}}{\delta z_{0}}$ vs. $\mathrm{t}$ plot gives the value of $\lambda$. The value of $\lambda$ in the present study is estimated using the function 'maxLyapunov', employing Equation (5) in the nonlinear series package of R software. Sample entropy (S) is another statistical tool based on information theory that measures the uncertainty in a time series. The correlation sum $\mathrm{C}(\mathrm{r})$ for a radius $\mathrm{r}$ and embedding dimension $\mathrm{m}$ and $\mathrm{m}+1$ is used to estimate the value of $\mathrm{S}$ using Equation (6) (Chen et al., 2019). The value of $\mathrm{S}$ for a particular dimension is its average value for different $r$. The 'sampleEntropy' function in the nonlinear series package of $\mathrm{R}$ is used to estimate $\mathrm{S}$ using Equation (6).

$S=\ln \left(\frac{C^{m}(r)}{C^{m+1}(r)}\right)$

For investigating the self-affinity of the breath sound signal, fractal analysis is done by estimating the fractal dimension (D) and Hurst exponent $(\mathrm{H})$. The signals originating from systems with complex dynamics also have a fractal nature (Mandelbrot, 1982). Box counting method, which counts the number of boxes occupied by the fractal pattern at different scales, is a simple and effective method for estimating D. In this method, the number of boxes $\mathrm{N}(\varepsilon)$ containing the signal at different box sizes $\varepsilon$ is counted. The slope of the $\log N(\varepsilon)$ vs. $\log \left(\frac{1}{\varepsilon}\right)$ plot gives the value of $\mathrm{D}$ (Gneiting et al., 2012). The package 'fractaldim' in the R software employs the function 'fd.estim.boxcount' is used to estimating D. The Hurst exponent $\mathrm{H}=2-$ $\mathrm{D}$ is calculated using the value of $\mathrm{D}$ (Kenkel et al., 1996). Depending on the value of $\mathrm{H}$, the time series is classified into (i) antipersistent $(0 \leq \mathrm{H}<0.5)$, (ii) random $(\mathrm{H} \sim 0.5)$ and (iii) persistent $(0.5<\mathrm{H} \leq 1)($ García \& Requena, 2019).

Principal component analysis (PCA) is a tool to decrease the dimensionality of a given dataset, conserving its variance for grouping into identical clusters. The PSD value is nearly equal for datasets with exact spectral features, enabling PSD to be a vital component for grouping. Features are selected by dividing the frequency range between $100 \mathrm{~Hz}$ and $1000 \mathrm{~Hz}$ into $n$ parts $(n=26)$ and finding the mean PSD value of each piece. The packages 'FactoMineR' and 'factoextra' in R software are employed for the PCA analysis using the extracted 26 features (Moses, 2015; Jolliffe \& Cadima, 2016; Husson et al., 2017). 
Computer-based automated classifiers are required to classify lung sounds rapidly in realtime. The accuracy of classification mainly depends on the selected method and features. The present work employs supervised MLTs like LDA, QDA, SVM, and KNN to classify BR and ST signals. For supervised MLTs, the phase portrait parameters such as D, S, $\lambda, l$ and $\mathrm{m}$, which represent the nonlinear properties of the movement, are selected as the variables or predictors. The accuracy and sensitivity of the classification are analyzed from the confusion matrix (CM). The CM gives an overall view of the actual values and predicted values by providing the four fundamental values - True Positive (TP), True Negative (TN), False Positive (FP), and False Negative (FN). The TP and TN represent the number of correctly predicted positive and negative values, respectively. The FP represents the number of negative values wrongly predicted as positive, and FN represents the number of positive values wrongly expected as unfavorable. Based on the values of TN, TP, FP, and FN from CM, the statistical parameters of MLT (accuracy, precision, specificity, sensitivity, and error rate) can be evaluated. A classification technique's accuracy (A) gives its overall effectiveness in giving exact predictions. A value can be assessed by dividing the number of samples predicted accurately by the entire pieces considered, as shown in Equation (7) (Amin et al., 2015; Swapna et al., 2021).

$A=\frac{(T P+T N)}{(T P+F P+T N+F N)}$

The true-positive rate (TPR) or sensitivity is the ratio of accurate positive predictions to the total positive cases. Equation (8) gives the value of TPR.

$T P R=\frac{T P}{T P+F N}$

The true-negative rate (TNR) or specificity gives the total number of accurate negative predictions relative to the actual total negatives (Equation (9)). The high value of sensitivity and specificity points to the high efficiency of an MLT on a particular data set. The total number of accurate positive predictions relative to the whole positive predictions is the positive predictive value (PPV) or precision of an MLT (Equation (10)). The inefficiency of an MLT can be evaluated by finding the error rate (ER) using Equation (11). The ER value represents the number of wrong predictions relative to the whole samples considered.

Along with the CM, the Receiver Operating Characteristic (ROC) curve also gives information about the effectiveness of MLT used. The area under the ROC plot measures distinguishability between the classes. The classification Learner app in the Matlab software is utilized to perform MLTs (Subasi, 2019).

$$
\begin{aligned}
& T N R=\frac{T N}{T N+F P} \\
& P P V=\frac{T P}{T P+F P} \\
& E R=\frac{(F P+T N)}{(T P+F P+T N+F N)}
\end{aligned}
$$




\section{Results and discussion}

Computerized digital auscultation and signal processing techniques are becoming a quantitative and accurate tool for diagnosing respiratory tract diseases. The time-series spectrum of the lung sound gives information about the lung condition buried in it. Among the 60 breath signals, BR and ST, representative signals are shown in Figures 1a and 1b, respectively, with a portion of each signal enlarged (Figures $1 \mathrm{c}$ and $1 \mathrm{~d}$ ). The duration of the inspiration and expiration phases is almost similar, and there is a distinct pause between the phases in the time domain signal of $\mathrm{BR}$ and ST. The loudness of ST and BR is due to the turbulent airflow through the largediameter region of the respiratory tract (Bohadana et al., 2014). In ST, the constricted area, due to lesion, introduces turbulence and modulation in the signal (Udupi \& Prabhakar,2016). The discontinuous, noisy nature of the BR is evident from Figure 1c. The musical and sinusoidal nature of the ST is visible in the magnified time-domain signal of ST (Figure 1d) (Barker \& Elphick, 2018). The time-domain signal of ST also contains regions, a combination of two modulated signals. The ST signal, continuing for more than $250 \mathrm{~ms}$ without interruption, reveals its inherent continuous nature (Andrès et al., 2018).

The turbulent flow of air through the respiratory tract with varying diameter produces a spectrum of frequencies, whose spread, intensity, and number of components gives valuable information about the condition of the airways. A representative normalized power spectral density (PSD) plot with an energy envelope, drawn using the FFT data, of the BR and ST during respiration is shown in Figure 2. Several frequency components of higher intensity are visible from the normalized PSD plot of BR and ST (Figure 2). The higher degree of freedom of the air passing through the trachea and upper airways of a larger cross-section is responsible for the number of frequency components in BR and ST. A comparison of Figures $2 a$ and $2 b$ show many frequency components of high intensity in BR spread over a limited frequency range of $200 \mathrm{~Hz}$ $(150 \mathrm{~Hz}$ to $350 \mathrm{~Hz})$. In the case of ST shown in Figure 2b, the span of frequency distribution ranges from $215 \mathrm{~Hz}$ to $580 \mathrm{~Hz}$, with very few frequency components of high intensity. The lesser number of high intense frequency components in ST agrees well with the literature report of its musical nature. The wider frequency band in ST can be attributed to the non-uniformity in the trachea and upper airways cross-sectional area due to the lesion (Udupi \& Prabhakar, 2016). The airflow velocity through the constricted regions is more significant than that of other standard regions due to the obstacles present. This increase in velocity increases the oscillations of the walls that account for the spreading frequency in ST.

The energy envelope shown in the normalized PSD plot gives information about energy distribution over the spectral range. The peaks of the energy envelope are labeled as A, B, and $\mathrm{C}$ in Figure 2. When BR shows a single dominant peak at $265 \mathrm{~Hz}$ (peak A) in the energy envelope plot, ST shows three significant peaks A, B, and C at $256 \mathrm{~Hz}, 369 \mathrm{~Hz}$, and $540 \mathrm{~Hz}$, respectively. The full width at half maximum of the peak A in BR is $82 \mathrm{~Hz}(224 \mathrm{~Hz}-305 \mathrm{~Hz})$, while it is $74 \mathrm{~Hz}(219 \mathrm{~Hz}-293 \mathrm{~Hz})$ in ST. The frequency range of peak A in ST suggests its origin is the same as in BR. In other words, ST comprises the signature of BR. From Figure 2b, it can be inferred that the appearance of peaks $\mathrm{B}$ and $\mathrm{C}$ at a higher frequency is due to the lesion in the trachea and upper airways. The signature peaks B and C of ST can be effectively used to monitor the lesion's level during treatment continuously. For understanding the type of ST, it is 
essential to analyze the inspiration and expiration separately. The normalized PSD plot of inspiration and expiration of BR and ST (Figure 3) is similar. P peaks B and C during inspiration and expiration of ST (Figures 3c and 3d) suggest that the type of stridor is biphasic due to the upper airway obstruction. In the case of inspiratory or expiratory stridors, peaks $\mathrm{B}$ and $\mathrm{C}$ arise only during the inspiration or expiration. Peak A appears invariably in all the three types of stridors, as in BR.
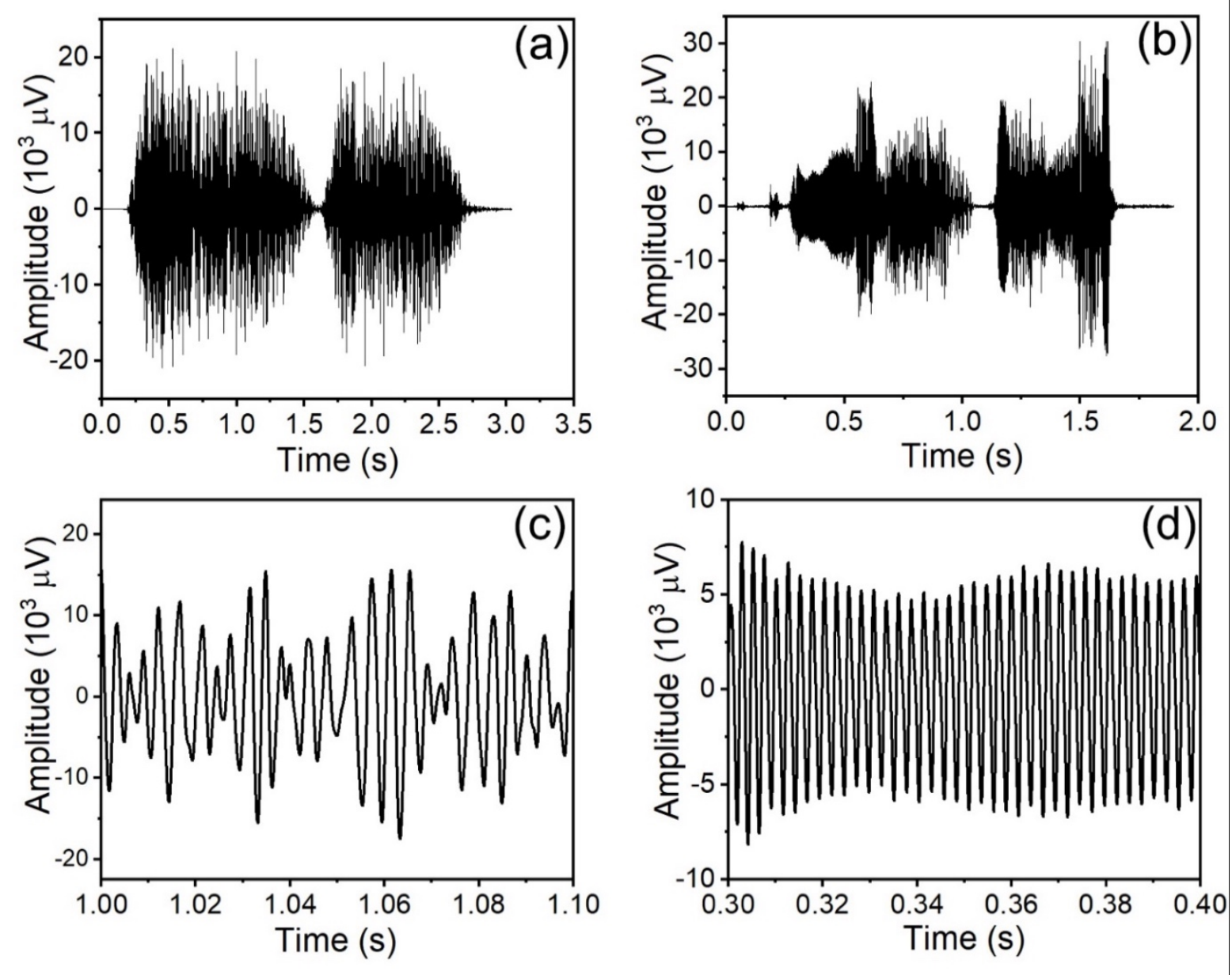

Fig. 1. Time-domain signal of (a) BR and (b) ST. The magnified time-domain signal of (c) BR and (d) ST shows musical nature. 


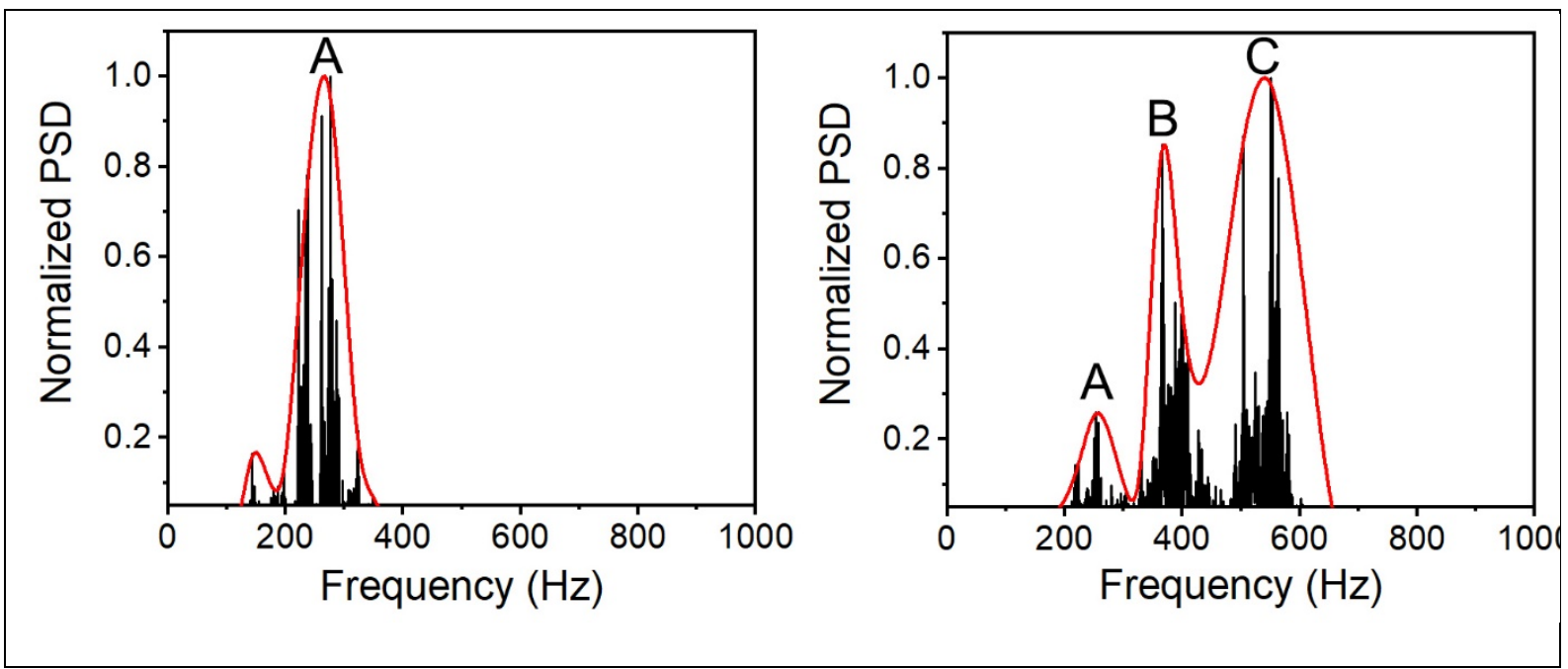

Fig. 2. The normalized PSD plot with energy envelope of (a) BR and (b) ST
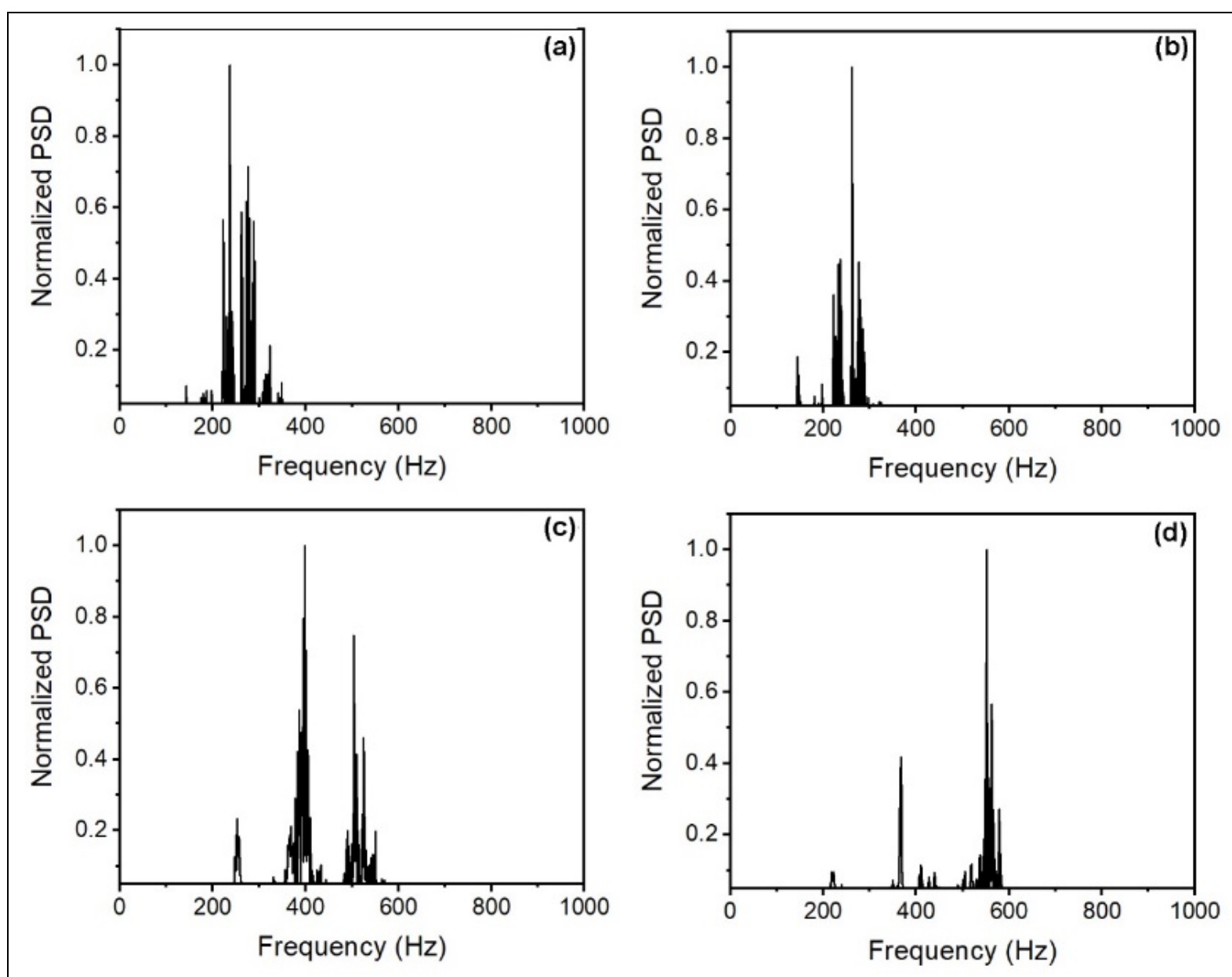

Fig. 3. The normalized PSD plot of (a) BR inspiration, (b) BR expiration, (c) ST inspiration, and (d) ST expiration.

The normalized PSD plot gives only the frequency components in the signal, whereas wavelet analysis provides information about the temporal evolution of the frequency components. The wavelet scalogram of the BR and ST are shown in Figure 4. The distinct pause 
between the inspiration and expiration for both the signals is visible in the wavelet scalogram (Bohadana et al., 2014). The wavelet scalogram of BR shown in Figure 4a shows the persistence of the dominant frequency, peak A, shown in Figure 2a, and the frequency spread during respiration. In the wavelet scalogram of ST, shown in Figure 4b, an increase with time is visible in the value of frequency and its magnitude, which can be interpreted using Bernoulli's principle. When air passes through a constricted region in ST, the pressure decreases, or the flow velocity increases in the flow direction. Such a temporal evolution of frequency and magnitude in the wavelet scalogram can be considered a signature of ST. As observed in Figure $2 \mathrm{~b}$, the increased frequency spread in ST is explained earlier.

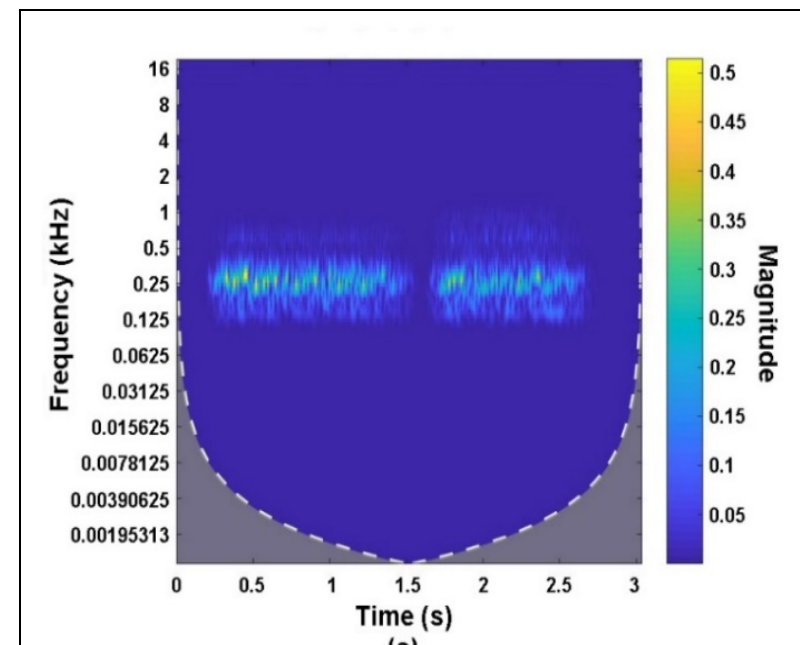

(a)

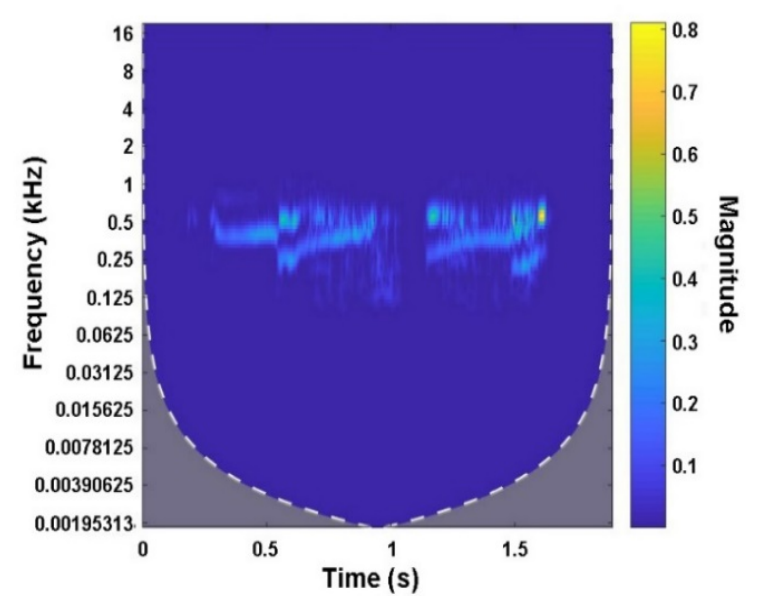

(b)

Fig. 4. Wavelet scalogram of (a) BR and (b) ST

The information about the complexity of the dynamics of a system can be understood by plotting its phase portrait. BR's phase portrait and three-dimensional representation are shown in Figures 5a and 5b. The phase portrait of BR shows a random distribution of densely packed phase points, the evolution of which is understood from the three-dimension plot. The randomness in phase points is revealed by plotting 4000 phase points of $\mathrm{BR}$, as shown in Figure 5c. The phase portrait of ST and its three-dimensional representation are shown in Figures $6 \mathrm{a}$ and $b$, respectively. The exciting patterns observed in the time spectra of ST (Figures $1 \mathrm{~b}$ and 1d) are also visible in the phase portrait. The signal contains a discontinuous region, musical and non-musical (with some kind of discontinuous modulation) part, whose phase portraits are separately investigated with 4000 phase points, as shown in Figures 6c, 6d, and 6e, with the respective time-domain signal in the inset. The phase portrait of the discontinuous region of ST, shown in Figure 6c, is identical to the phase portrait of BR (Figure 5c) due to peak A with multiple frequency components in both BR and ST. Figure 6d shows an ordered phase portrait of almost elliptical shape for the continuous musical region of the time domain signal of ST. This ordered phase portrait is due to the musical nature of ST, as evidenced by the PSD plot. The ST signal exhibiting regions of superimposition of multiple signals also gets reflected in its phase portraits as elliptical trajectories of different eccentricities, as shown in Figure 6e. 


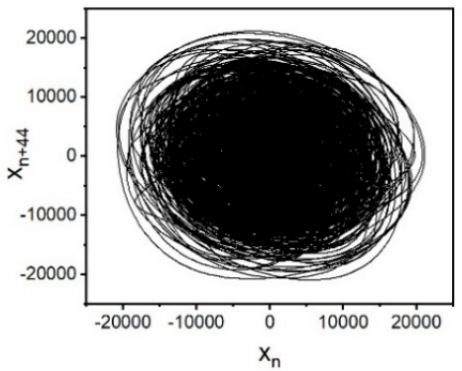

(a)

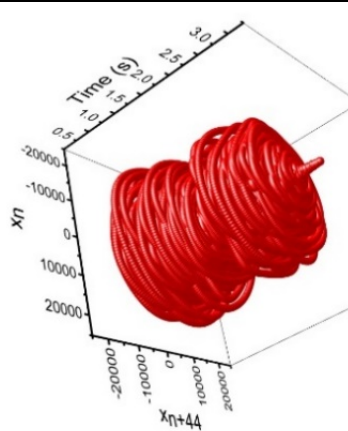

(b)

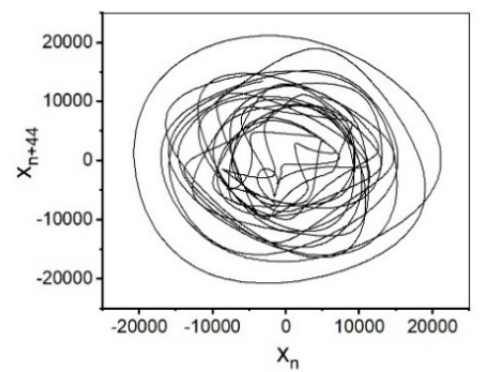

(c)

Fig. 5. Phase portrait of BR (a) two-dimensional representation, (b) three-dimensional representation, and (c) with 4000 data points

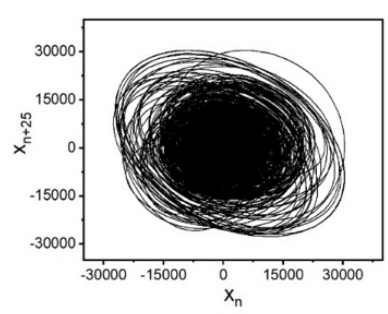

(a)

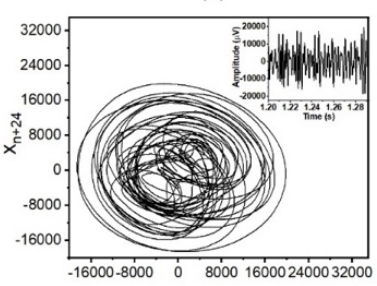

$x_{n}$

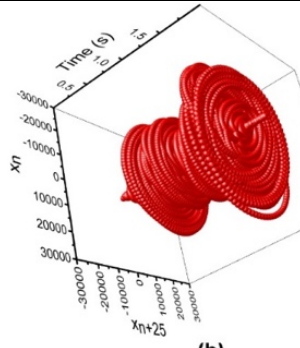

(b)

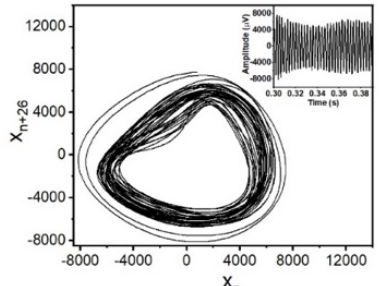

(d)

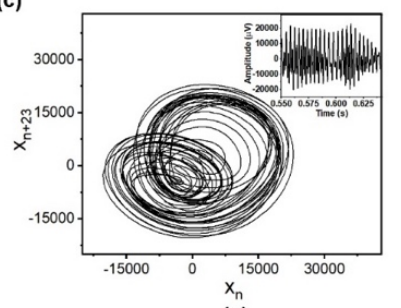

(e)

Fig. 6. Phase portrait of ST (a) two-dimensional representation, (b) three-dimensional representation; with 4000 points unveiling its characteristics along with the time domain signal in the inset (c) discontinuous, (d) musical, and (e) non-musical 
Table 1. Fractal dimension, Hurst exponent, and sample entropy of BR and ST

\begin{tabular}{ccc}
\hline Parameter & BR & ST \\
\hline Fractal dimension & $1.809 \pm 0.003$ & $1.739 \pm 0.006$ \\
Hurst exponent & $0.191 \pm 0.003$ & $0.261 \pm 0.006$ \\
Sample entropy & $1.28 \pm 0.10$ & $0.9187 \pm 0.03$ \\
\hline
\end{tabular}

The fractal analysis of self-affine time-series signals is vital for computing its complexity. In the present study, the Box-counting method is employed to estimate the fractal dimension (D) of BR and ST, from which the Hurst exponents $(\mathrm{H})$ are calculated. The values of D and H are given in Table 1. The higher value of $\mathrm{D}$ of $\mathrm{BR}$ can be thought of as originating from the discontinuity and the presence of high intense frequency components (peak A in Figure 2a), as evidenced by the PSD plot. The continuous nature of the ST with a particular dominant frequency component makes the time-series signal of ST less complex relative to BR. The values of $\mathrm{H}$ given in Table 1 , between 0 and 0.5 , indicate the antipersistence nature of the $\mathrm{BR}$ and ST. The antipersistence nature of BR is higher than ST as the value of $\mathrm{H}$ of $\mathrm{BR}$ is closer to zero.

The PSD and wavelet analyses show the signature of BR in ST, which is investigated from the portions of the time domain signal of ST, exhibiting different features like discontinuity, musical and non-musical, as presented in Figures 6c to 6e. The Maximal Lyapunov exponent $(\lambda)$ calculated with the phase points represented in Figures $6 \mathrm{c}$ to $6 \mathrm{e}$ is obtained as $0.252,0.086$, and 0.137 , respectively. The analysis reveals that the temporal evolution of divergence $\lambda$ is the least in the musical region compared to the other two areas. The disorder of a time series can be quantified by estimating its sample entropy. The value of the sample entropy $(\mathrm{S})$ of the BR and $\mathrm{ST}$ is shown in Table 1. The higher value of $\mathrm{S}$ for BR is evident from Table 1, which points to its disordered nature. The value of $\mathrm{S}$ for the phase points represented in Figures $6 \mathrm{c}$ to $6 \mathrm{e}$ are $2.36,0.762$, and 1.98 , respectively. The lowest $\mathrm{S}$ value for Figure $6 \mathrm{~d}$, the musical region, clearly reveals its more ordered behavior.

Principal component analysis (PCA) is a dimensionality reducing technique used to classify and group signals. Here the frequency region $100 \mathrm{~Hz}$ to $1000 \mathrm{~Hz}$ in PSD data is divided into 26 segments, and the mean of PSD of each piece is taken as a characteristic feature. As a result, the mean value of the distinctive feature of each part $(\mu)$ of BR and ST, represented in Figure 7 , is distinct, enabling the grouping in the PCA. For example, the peak value of $\mu$ for BR appears in peak $\mathrm{A}$, whereas for ST, the peaks $\mathrm{A}, \mathrm{B}$, and $\mathrm{C}$ are distinctively visible. The PCA carried out using the extracted features shows $80.2 \%$ of the total variance in data in the principal component 1 and 2. Figure 8 shows the plot of main components 1 and 2, clear separation between the points of BR and ST. 


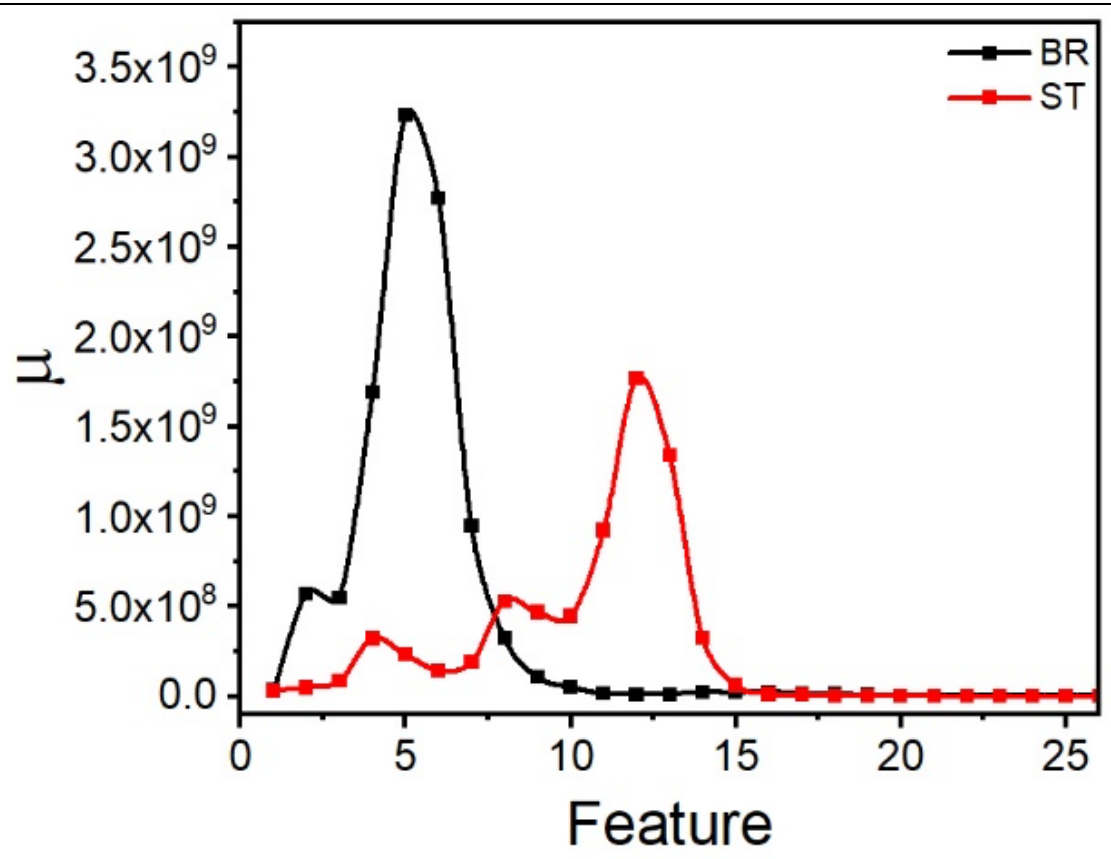

Fig. 7. The variation of $\mu$ with feature number for BR and ST

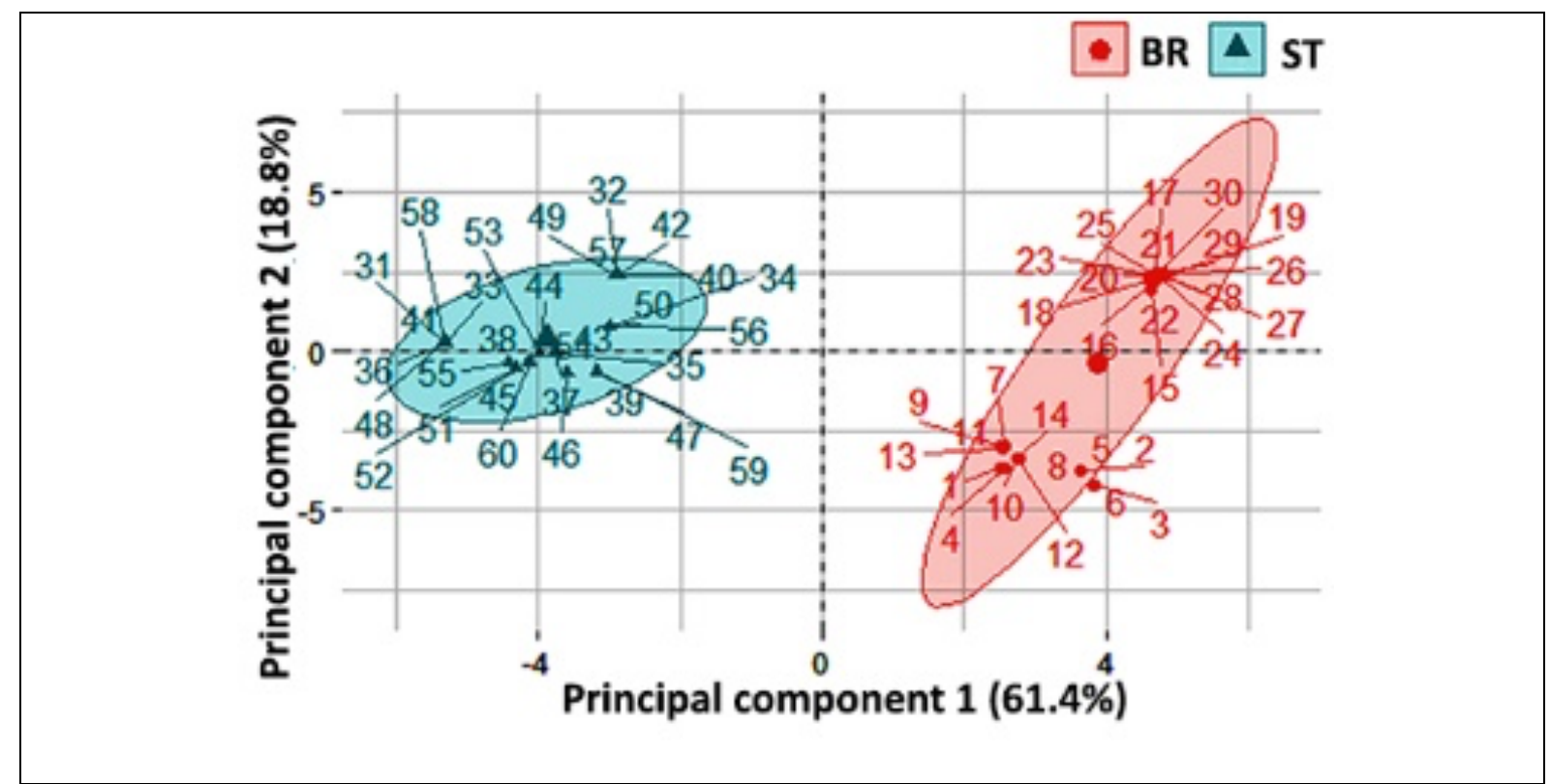

Fig. 8. PCA plot of BR and ST

Though PCA helps in classifying the signals by finding the directions of the highest variance, it has the limitation that it is unsupervised. This limitation is overcome by using supervised MLTs such as LDA, QDA, SVM, and KNN, which offer high specificity, accuracy, sensitivity, and precision. For understanding the effectiveness of the phase portrait parameters in classifying ST and BR, D, S, $\lambda, l$ and $\mathrm{m}$ are selected as features in supervised MLTs. Among the 60 signals, 45 randomly chosen signals are selected for training the model. Five-fold crossvalidation is used to prevent overfitting in the model trainer. The performance of different 
MLTs in classifying BR and ST breath signals is shown in Table 2. From Table 2, it is clear that most of the MLTs employed in the present work show $100 \%$ accuracy, sensitivity, specificity, and precision, which points to the potential of $\mathrm{D}, \mathrm{S}, \lambda, l$, and $\mathrm{m}$ parameters in differentiating adventitious lung sound signal from normal ones. The accuracy of classification using QDA is higher than that of LDA. This higher accuracy might be because, unlike LDA, QDA assumes a different covariance matrix for another class, which increases the flexibility of QDA and enables it to classify the categories nonlinearly using quadratic decision boundaries. The confusion matrix of both LDA and QDA classifiers is shown in Figure 9, and its corresponding ROC curve is given in Figure 10. The area under the ROC curve is higher for QDA compared to LDA, which .indicates maximum distinguishability between classes in QDA.

In the case of the SVM classifier, the quadratic, cubic, medium gaussian, and coarse Gaussian kernel functions offer 100\% accuracy compared to others. Thus these SVM kernel functions are suitable for classifying nonlinear time series parameters as features. The selection of the number of neighbors $(\mathrm{k})$ is essential in the KNN classifier. From Table 2, it can be understood that the $\mathrm{KNN}$ classifiers with $\mathrm{k}=10$ show $100 \%$ accuracy, irrespective of their distance metric and weight, compared to others. The trained, supervised MLT models predict the class of 15 randomly selected breath sound signals in Matlab software. All the models except Coarse KNN predict the courses with 100\% accuracy. The confusion matrix of the predicted values using the Quadratic SVM model is shown in Figure 11. The highly accurate predictions by the modeled MLTs using phase portrait parameters can undoubtedly classify the lung sound of adventitious stridor from the normal one.

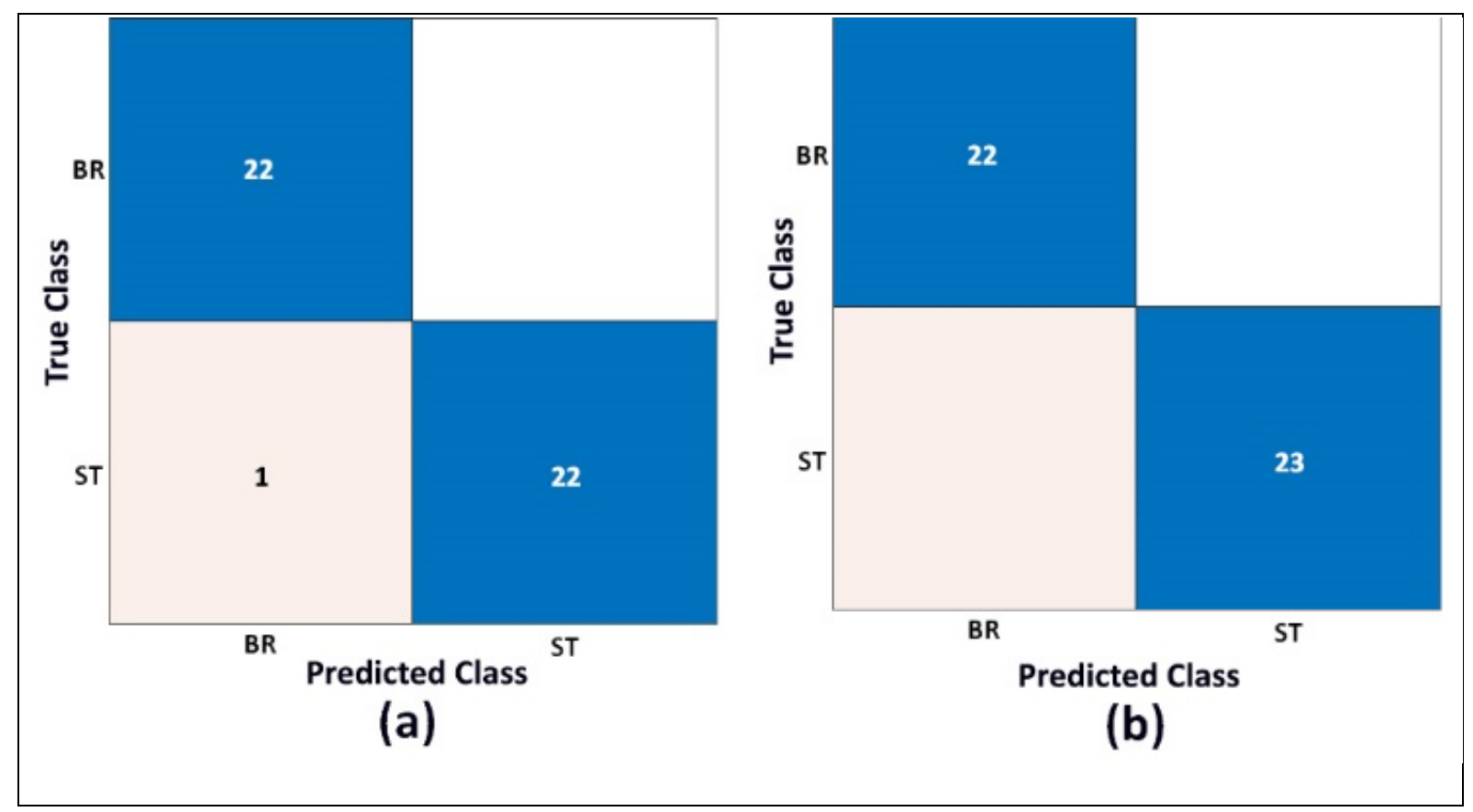

Fig. 9. Confusion matrix of classification - Training of dataset. (a) LDA and (b) QDA 


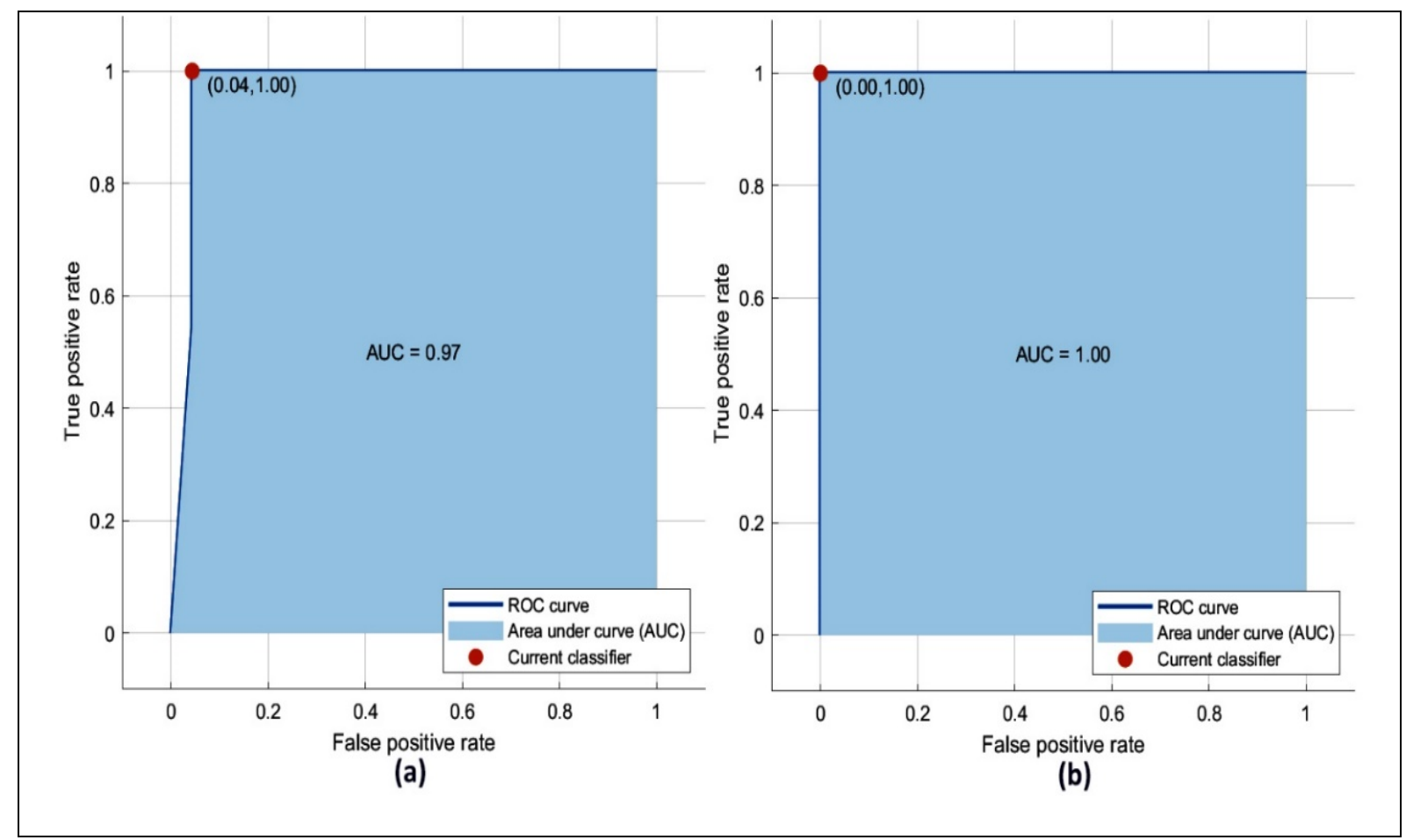

Fig. 10. Receiver Operating Characteristic curve - Training of dataset.

(a) LDA and (b) QDA

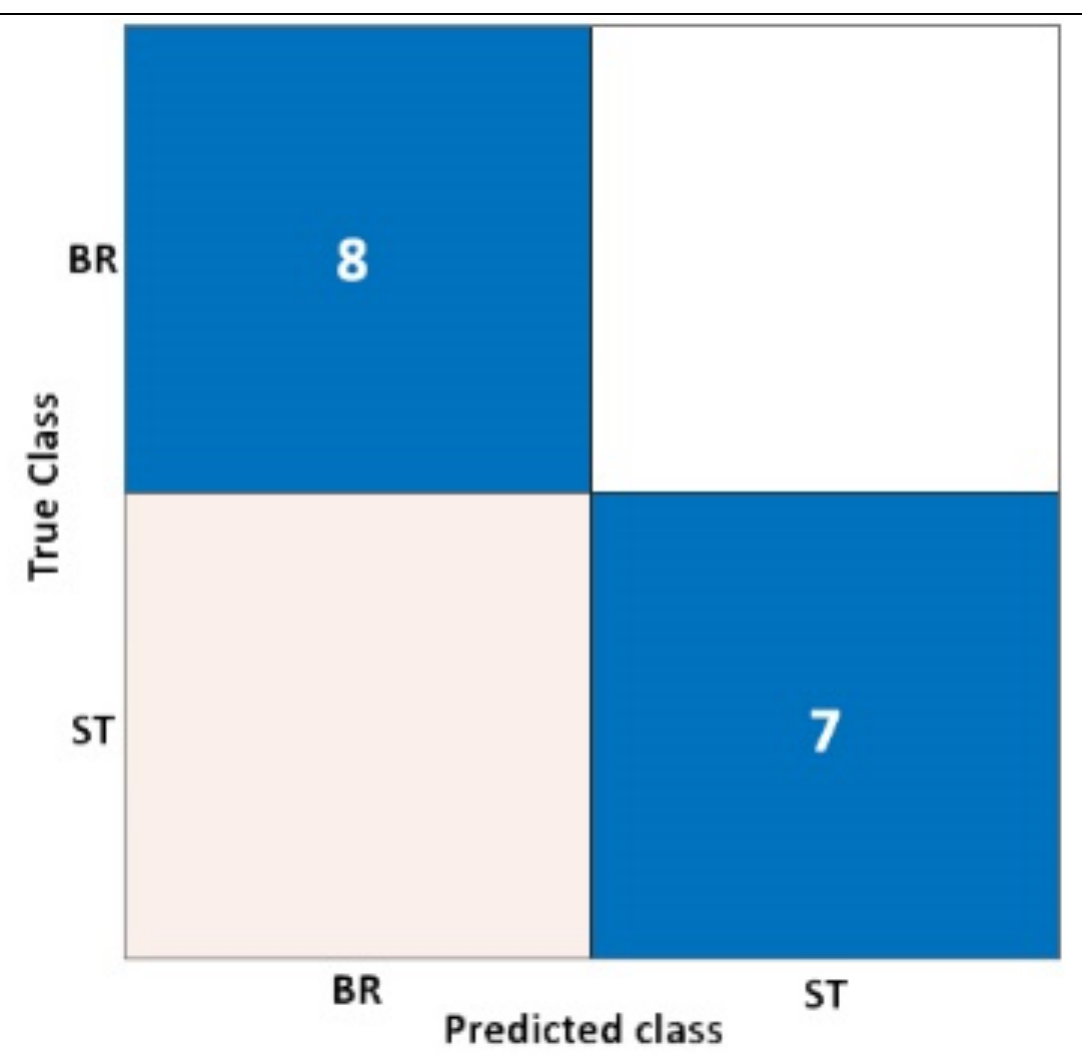

Fig. 11. Confusion matrix of prediction - Testing of the dataset using Quadratic SVM model 
Table 2. Performance of different MLTs in classifying BR and ST breath signal

\begin{tabular}{|c|c|c|c|c|c|c|}
\hline Model name & $\mathrm{K}$ & $\begin{array}{l}\text { Accuracy } \\
(\%)\end{array}$ & $\begin{array}{l}\text { Sensitivity } \\
(\%)\end{array}$ & $\begin{array}{l}\text { Specificity } \\
(\%)\end{array}$ & $\begin{array}{l}\text { Precision } \\
(\%)\end{array}$ & $\begin{array}{l}\text { Error rate } \\
(\%)\end{array}$ \\
\hline LDA & & 97.78 & 95.65 & 100.00 & 100.00 & 2.22 \\
\hline Q & & 100.00 & 100.00 & 100.00 & 100.00 & 0 \\
\hline \multicolumn{7}{|l|}{ SVM } \\
\hline Linear & & 97.78 & 95.65 & 100.00 & 100.00 & 2.22 \\
\hline Quadratic & & 100.00 & 100 & 100.00 & 100.00 & 0 \\
\hline Cubic & & 100.00 & 100 & 100.00 & 100.00 & 0 \\
\hline Fine Gaussian & & 82.22 & 79.17 & 85.71 & 86.36 & 17.78 \\
\hline Medium Gaussian & & 100.00 & 100 & 100.00 & 100.00 & 0 \\
\hline Coarse Gaussian & & 100.00 & 100 & 100.00 & 100.00 & 0 \\
\hline \multicolumn{7}{|l|}{$\mathrm{KNN}$} \\
\hline Fine & 1 & 95.56 & 95.45 & 95.65 & 95.45 & 4.44 \\
\hline Medium & 10 & 100.00 & 100 & 100.00 & 100.00 & 0 \\
\hline Coarse & 100 & 44.44 & 44.44 & 44.44 & 54.55 & 55.56 \\
\hline Cosine & 10 & 100.00 & 100 & 100.00 & 100.00 & 0 \\
\hline Cubic & 10 & 100.00 & 100 & 100.00 & 100.00 & 0 \\
\hline Weighted & 10 & 100.00 & 100 & 100.00 & 100.00 & 0 \\
\hline
\end{tabular}

\section{Conclusion}

Breath sounds carry vital information about the condition of the respiratory tract and lungs. Auscultation of breath sound with digital techniques makes the diagnosis of respiratory diseases more simple, accurate, reliable, and faster. Sixty regular BR and adventitious ST, produced from the trachea and upper airways, are investigated in the present study using spectral, nonlinear time series, and fractal analyses. A periodic continuous region in the time domain signal of ST spanning more than $250 \mathrm{~ms}$ suggests its musical nature. The spectral analysis of BR and ST using PSD plot and wavelet scalogram reveals the spread in frequency components of ST due to the obstructions in the trachea and upper airways caused by lesions in ST. The energy envelope on the normalized PSD plot has two signature peaks in ST (peak B and C) in addition to the expected peak A in BR and ST. The temporal evolution of the frequency and intensity in the wavelet of ST towards higher values suggests the increase in the velocity of air passing through the constricted regions. Nonlinear time series analysis is an example of the nonlinear signal processing technique used to understand airflow dynamics through the trachea 
and upper airways. The geometric representation of the dynamics revealed through the phase portrait analysis is random and dense for BR. The musical region of the ST has an ordered phase portrait, which seems to be superimposed with the random phase portrait of the discontinuous area.

The Fractal dimension of BR is higher than ST due to the intermittent nature and presence of multiple frequency components in BR. The Hurst exponent derived from the fractal dimension suggests the antipersistence nature of both BR and ST. The musical region's ordered nature is evidenced by its low sample entropy value and maximal Lyapunov exponent. The unsupervised and supervised MLTs classify BR and ST lung sounds. The unsupervised PCA analysis using the extracted features from the PSD plot clusters the BR and ST distinctively. Thus the study suggests the potential of the spectral, fractal, and nonlinear time series analyses in identifying ST, facilitating pulmonary auscultation. The signals are also classified with the phase portrait parameters D, S, $\lambda, l$, and $\mathrm{m}$ using supervised MLTs like LDA, QDA, SVM, and KNN, which classify the signals and help in prediction. The $100 \%$ predictability and accuracy shown by supervised MLTs suggest that these methods can effectively be used in the auscultation of breath signals.

\section{Conflict of interest}

The authors do not have any potential conflicts of interest to declare.

\section{References}

Ahlstrom, C., Johansson, A., Hult, P., \& Ask, P. (2006) Chaotic dynamics of respiratory sounds. Chaos, Solitons \& Fractals, 29(5):1054-1062

Amin, H. U., Malik, A. S., Ahmad, R. F., Badruddin, N., Kamel, N., Hussain, M., \& Chooi, W.-T. (2015) Feature extraction and classification for EEG signals using wavelet transform and machine learning techniques. Australasian Physical \& Engineering Sciences in Medicine, 38(1):139-149

Andrès, E., Gass, R., Charloux, A., Brandt, C., \& Hentzler, A. (2018) Respiratory sound analysis in the era of evidence-based medicine and the world of medicine 2.0. Journal of pharmaceutical and life, 11(2):89-106

Bahoura, M. (2009) Pattern recognition methods applied to respiratory sounds classification into normal and wheeze classes. Computers in Biology and Medicine, 39(9):824-843

Barker, N., \& Elphick, H. (2018) Respiratory Sounds: Laryngeal Origin Sounds. In Breath Sounds (pp. 237-247). Springer International Publishing, Cham.

Bohadana, A., Izbicki, G., \& Kraman, S. S. (2014) Fundamentals of Lung Auscultation. New England Journal of Medicine, 370(8):744-751

Cao, L. (1997) Practical method for determining the minimum embedding dimension of a scalar time series. Physica D: Nonlinear Phenomena, 110(1-2):43-50 
Chen, C., Sun, S., Cao, Z., Shi, Y., Sun, B., \& Zhang, X. D. (2019) A comprehensive comparison and overview of R packages for calculating sample entropy. Biology Methods and Protocols, 4(1)

Colak, C., Çolak, M. C., Ermiş, N., Erdil, N., \& Özdemir, R. (2016) Prediction of cholesterol level in patients with myocardial infarction based on medical data mining methods. Kuwait Journal of Science, 43(3):86-90

Debbal, S. M., \& Bereksi-Reguig, F. (2008) Computerized heart sounds analysis. Computers in Biology and Medicine, 38(2):263-280

Easyauscultation (2021) www.easyauscultation.com.

https://www.easyauscultation.com/cases?coursecaseorder=1\&courseid=201 (accessed March 27, 2021)

EMTprep (2021) www.EMTprep.com. https://emtprep.com/free-training/video/lung-soundscollection (accessed March 27, 2021)

García, M. de las N. L., \& Requena, J. P. R. (2019) Different methodologies and uses of the Hurst exponent in econophysics. Estudios de economía aplicada, 37(2):96-108

Gavriely, N., Palti, Y., \& Alroy, G. (1981) Spectral characteristics of normal breath sounds. Journal of Applied Physiology, 50(2):307-314

Gneiting, T., Ševč́ková, H., \& Percival, D. B. (2012) Estimators of Fractal Dimension: Assessing the Roughness of Time Series and Spatial Data. Statistical Science, 27(2):247-277

Gray, L., Denneny, J. C., Carvajal, H., \& Jahrsdoerfer, R. (1985) Fourier analysis of infantile stridor: preliminary data. International Journal of Pediatric Otorhinolaryngology, 10(2):191-199

Gurung, A., Scrafford, C. G., Tielsch, J. M., Levine, O. S., \& Checkley, W. (2011) Computerized lung sound analysis as a diagnostic aid for detecting abnormal lung sounds: A systematic review and meta-analysis. Respiratory Medicine, 105(9):1396-1403

Husson, F., Lê, S., \& Pagès, J. (2017) Exploratory multivariate analysis by example using R. CRC Press, Florida.

Jolliffe, I. T., \& Cadima, J. (2016) Principal component analysis: a review and recent developments. Philosophical Transactions of the Royal Society A: Mathematical, Physical and Engineering Sciences, 374(2065):20150202

Jones, A. (1995) A Brief Overview of the Analysis of Lung Sounds. Physiotherapy, 81(1):3742

Kantz, H., \& Schreiber, T. (2003) Nonlinear Time Series Analysis. Cambridge University Press, Cambridge.

Kenkel, N. C., \& Walker, D. J. (1996) Fractals in the biological sciences. Coenoses, 11(2):77100 
Leiberman, A., Cohen, A., \& Tal, A. (1986) Digital signal processing of stridor and snoring in children. International Journal of Pediatric Otorhinolaryngology, 12(2):173-185

Li, X., Cao, X., Guo, M., Xie, M., \& Liu, X. (2020) Trends and risk factors of mortality and disability-adjusted life-years for chronic respiratory diseases from 1990 to 2017: a systematic analysis for the Global Burden of Disease Study 2017. BMJ, m234

Mandelbrot, B. B. (1982) The fractal geometry of nature (Vol. 1). WH freeman New York, United States.

Medzcool (2021) https://www.medzcool.com/. https://www.medzcool.com/ (accessed March 27, 2021).

Monto, A. S., \& Fukuda, K. (2020) Lessons From Influenza Pandemics of the Last 100 Years. Clinical Infectious Diseases, 70(5):951-957

Moses, D. (2015) A survey of data mining algorithms used in cardiovascular disease diagnosis from multi-lead ECG data. Kuwait Journal of Science, 42(2):206-235

Noble, W. S. (2006) What is a support vector machine? Nature Biotechnology, 24(12):15651567

Pfleger, A., \& Eber, E. (2016) Assessment and causes of stridor. Pediatric Respiratory Reviews, 18:64-72

Plums, W. A., van Mook, W. N., Wittekamp, B. H., \& Bergmans, D. C. (2015) Postextubation laryngeal edema and stridor resulting in respiratory failure in critically ill adult patients: an updated review. Critical Care, 19(1):295

Polat, H., \& Güler, İ. (2004) A Simple Computer-Based Measurement and Analysis System of Pulmonary Auscultation Sounds. Journal of Medical Systems, 28(6):665-672

Pramono, R. X. A., Bowyer, S., \& Rodriguez-Villegas, E. (2017) Automatic adventitious respiratory sound analysis: A systematic review. (T. Penzel, Ed.)PLOS ONE, 12(5):e0177926

Raj, V., Swapna, M. S., Satheesh Kumar, K., \& Sankararaman, S. (2020) Temporal evolution of sample entropy in the thermal lens system. Chaos: An Interdisciplinary Journal of Nonlinear Science, 30(4):043113

Sarkar, M., Madabhavi, I., Niranjan, N., \& Dogra, M. (2015) Auscultation of the respiratory system. Annals of Thoracic Medicine, 10(3):158

Subasi, A. (2019) Practical guide for biomedical signals analysis using machine learning techniques: A MATLAB based approach. Academic Press, Cambridge, United States.

Swapna, M. S., Sreejyothi, S., \& Sankararaman, S. (2020) Investigation of fractality and variation of fractal dimension in germinating seed. The European Physical Journal Plus, 135(1):38

Swapna, M. S., Sreejyothi, S., Renjini, A., Raj, V., \& Sankararaman, S. (2021) Unravelling the potential of phase portrait in the auscultation of mitral valve dysfunction. The European Physical Journal Plus, 136(2):184 
Udupi B. P., \& Prabhakar, H. (2016) Stridor. In Complications in Neuroanesthesia (pp. 237246). Elsevier, Amsterdam.

Wallace, J. C., Raaphorst, G. P., Somorjai, R. L., Ng, C. E., Fung Fung, M. K, Senterman, M., \& Smith, I. P. (1997) Classification of1H MR spectra of biopsies from untreated and recurrent ovarian cancer using linear discriminant analysis. Magnetic Resonance in Medicine, 38(4):569-576

Submitted: $\quad 11 / 12 / 2020$

Revised: $\quad 28 / 03 / 2021$

Accepted: $\quad 14 / 04 / 2021$

DOI: $\quad 10.48129 / \mathrm{kjs} .11363$ 